\title{
CREO: EL ASCENSO Y LOS DESAFÍOS DE CONSOLIDACIÓN DEL PARTIDO POLÍTICO DE DERECHA EMERGENTE EN ECUADOR (2013-2021) ${ }^{1}$
}

CREO: the rise and consolidation challenges of the emerging rightwing political party in Ecuador (2013-2021)

\author{
Patricio Navia \\ New York University / Universidad Diego Portales \\ patricio.navia@nyu.edu \\ https://orcid.org/0000-0001-9398-8393
}

\section{Sebastián Umpiérrez de Reguero}

Universidad Diego Portales / Leiden University / European University Institute sebastian.umpierrez@mail.udp.cl https://orcid.org/0000-0002-5464-0690

Recibido: $1 / 2 / 2021$

Aceptado: 14/5/2021

Resumen: Este artículo analiza los desafíos de ascenso y consolidación del partido de derecha actualmente más grande de Ecuador, a nivel nacional, el Movimiento Creando Oportunidades (CREO). Nuestro enfoque se centra en el efecto de las reglas electorales y la organización interna de CREO. Empleando un análisis de oferta-demanda, discutimos las razones detrás de su éxito electoral de 2013 a 2021. Describimos su desempeño electoral por tipos de elecciones y género de candidatos, así como por provincias y distritos en el extranjero. En general, CREO ha surgido y evolucionado en parte opacado por el correísmo (antes Movimiento Patria Altiva I Soberana [MPAIs], ahora Revolu-

1 Los autores agradecen la retroalimentación de los dos revisores de pares ciegos, así como a Felipe Monestier, Gabriel Vommaro, Régis Dandoy y Germán Campos Herrera por sus comentarios en versiones previas. 
ción Ciudadana $[\mathrm{RC}]$ ), pero logró convertirse en un serio competidor electoral nacional desde 2017 y ganar la presidencia en 2021. Después de las elecciones de abril de 2021, sus principales desafíos radican en la dependencia de la figura de Guillermo Lasso y su continuidad como principal partido de derecha en el futuro en el ámbito nacional.

Palabras clave: partido de derecha, reglas electorales, organización interna, anticorreísmo, Ecuador.

Abstract: This article analyzes the rise and consolidation challenges of the largest right-wing party in Ecuador at the national level: Movimiento Creando Oportunidades (CREO). Our approach focuses on the effect of electoral rules and CREO's internal organization. Employing supply and demand analyses, we discuss the rationale behind its electoral success from 2013 to 2021. Thereafter, we outline CREO's electoral performance per types of elections and candidate gender, as well as by provinces and overseas districts. Overall, CREO has emerged and evolved overshadowed by the Movimiento Patria Altiva I Soberana (MPAIS), particularly from Correism, to become a serious national electoral competitor since 2017 and won the presidential office in 2021. After the latest elections on April 2021, its main challenges stem from the dependence of the figure of Guillermo Lasso and its continuity as main right-wing party in the future.

Keywords: right-wing party, electoral rules, internal organization, anti-correism, Ecuador. 


\section{Introducción}

Ecuador es uno de los pocos países de América Latina que ha visto el surgimiento de un poderoso partido de derecha desde la oposición en los últimos años. Si bien a nivel de construcción partidaria ${ }^{2}$ CREO no es aún un caso exitoso (acorde con los criterios de Levitsky, Loxton y Van Dyck, 2016), tiene serias posibilidades de serlo. Fundado en 2013, el Movimiento Creando Oportunidades (CREO) es el partido de derecha más grande del país. Obtuvo más del $48 \%$ de los votos en la segunda vuelta de las elecciones presidenciales de 2017 , la elección presidencial más competitiva desde 1998, y en 2021, ganó la presidencia (alrededor del $52 \%$ de los votos) frente al candidato correísta, Andrés Arauz. En sus poco más de siete años de existencia, CREO se convirtió en un actor importante en la política ecuatoriana. Después de recibir menos votos que el Partido Social Cristiano (PSC) en las elecciones locales y regionales de 2019, CREO retomó su lucha anticorreísta, aliándose al PSC para las elecciones presidenciales de 2021. Como está fuertemente asociado a su líder fundador, Guillermo Lasso, CREO corre el riesgo de depender del desempeño y aprobación de Lasso como presidente y de las aspiraciones futuras de su líder. El partido puede consolidarse como un partido nacional de derecha fuerte si construye un apoyo electoral sostenible, independientemente de la administración de Lasso.

Este capítulo analiza el ascenso de CREO, y busca explicar su consolidación como el partido de derecha más grande de Ecuador. Examinamos el desempeño electoral de CREO en elecciones nacionales, regionales y locales. Desde su fundación, CREO ha participado en tres procesos electorales nacionales y supranacionales, así como en dos comicios locales y regionales (anexo 1). CREO se convirtió en el partido de oposición más importante durante el segundo mandato presidencial de Rafael Correa (2013-2017) y se alió estratégicamente al gobierno de Lenín Moreno (2017-2021) para poner freno a las ambiciones de Correa de mantenerse en el poder después de dejar la presidencia en 2017. Mientras realizaba una campaña activa contra los esfuerzos del correísmo para abolir los límites del mandato presidencial en 2014-2015, CREO se estableció como un partido comprometido con las instituciones democráticas y las políticas favorables al mercado. A nivel nacional, se convirtió rápidamente en el segundo partido electoral más grande del país, después del Movimiento Patria Altiva I Soberana (MPAIS), el partido inicialmente formado por Correa. Sin embargo, tuvo reveses como la ruptura de su alianza preelectoral con Sociedad Unida Más Acción (suMA) después de las

2 Levitsky, Loxton y Van Dyck (2016, p. 4) definen la construcción partidaria como «el proceso por el cual los nuevos partidos se convierten en actores políticos duraderos y significativos desde el punto de vista electoral». 
elecciones de 2017, que hicieron que el voto se fragmente aún más entre las fuerzas de derecha en las elecciones seccionales de 2019. En las últimas elecciones nacionales estas alianzas y tensiones con otros partidos de derecha se tradujeron en una diferencia negativa en escaños legislativos con respecto a 2017, y a casi no pasar a la segunda vuelta en 2021.

CREO se beneficia de la relativa popularidad de su líder y contendiente presidencial en tres ocasiones (2013, 2017 y 2021): Guillermo Lasso. En las elecciones de 2017, Lasso ganó en varias circunscripciones electorales y estuvo cerca de derrotar al candidato de MPAIS, Lenín Moreno, elegido originalmente como sucesor de Correa. A pesar de perder el balotaje, Lasso recibió más votos que Moreno en 13 de las 24 provincias de Ecuador, así como 2 de las 3 circunscripciones del exterior. Un resultado muy similar se puede observar en 2021, cuando de manera algo sorpresiva, Lasso logró triunfar en la segunda vuelta de la elección presidencial.

Antes de presentar un resumen de la historia e ideología de CREO, discutimos las posibles razones detrás de su éxito electoral (2013-2021). Abordamos el desempeño electoral de CREO como un análisis de oferta y demanda, centrándonos en la influencia de las reglas electorales, el contexto político de Ecuador, y la organización interna de CREO. ${ }^{3}$ En las siguientes secciones, revisamos descriptivamente el desempeño electoral de CREO y resumimos nuestros hallazgos, además, especulamos sobre los desafíos que enfrenta para consolidarse como un partido nacional independiente más allá del éxito en las presidenciales de 2021.

\section{Las razones del ascenso electoral de CREO}

Alineados con la literatura sobre construcción partidaria (Levitsky, Loxton, Van Dyck, 2016; Rosenblatt, 2018; Vommaro, 2019) discutimos las condiciones exógenas que probablemente han impulsado el surgimiento de una organización de derecha relativamente nueva: CREO. En particular, examinamos lo que hizo este partido - que otras organizaciones políticas de derecha de facto no hicieron- para emerger como el principal partido de derecha en Ecuador. Analizamos el lado de la demanda y el lado de la oferta para vislumbrar el auge de CREO en la política ecuatoriana.

3 La organización partidaria la definimos como la estructura, pero también los recursos —incluidas las redes y capital humano-, que tiene el partido para operar en un contexto democrático (Webb, Scarrow y Poguntke, 2019). 


\subsection{Lado de la demanda}

A principios de la década de 2000, los partidos tradicionales de la derecha ecuatoriana estaban en decadencia (Machado Puertas, 2007; Mainwaring, 2006). Al igual que en otros países de la región donde el sistema de partidos colapsó o se debilitó aún más tras el paso del siglo (Carreras, 2012; Cyr, 2016; Seawright, 2012), este declive en Ecuador se acentuó con el ascenso a la presidencia del líder carismático de izquierda Rafael Correa en 2007 (Machado Puertas, 2008). Correa, un candidato inconformista que, pese a una corta carrera en el sector público, logró vencer a candidatos con más experiencia política en varias elecciones nacionales, redefiniendo la arena política en Ecuador (Basabe-Serrano, Pachano y Mejía Acosta, 2010; Wolff, 2018).

Correa fundó MPAIS en 2006. Ese mismo año ganó la segunda vuelta de las elecciones presidenciales al derrotar al candidato de derecha Álvaro Noboa por un margen de 13,3\% (CNE, 2014). Como candidato y en sus primeros años en el gobierno, Correa contó con el apoyo de movimientos sociales indígenas y sindicatos (Collins, 2014). Forjó un estilo de gobierno estrechamente relacionado con la ideología del socialismo del siglo xxI y una estrategia que se puede calificar como campaña permanente (Barr, 2017; Basabe-Serrano y Martínez, 2014; Conaghan y De La Torre, 2008; Polga-Hecimovich, 2013). Tras convocar una asamblea constituyente que redactó una nueva constitución en 2007-2008, ganó nuevas elecciones presidenciales en 2009 y 2013 (CNE, 2014). Correa extendió sus reformas a áreas como el sistema judicial y los medios de comunicación, aumentó el poder del Estado y concentró el poder en manos del presidente (Freidenberg, 2012; Montúfar, 2016).

Durante los dos primeros mandatos de Correa (2007-2013), Ecuador experimentó una bonanza económica provocada por un repunte internacional de los precios del petróleo y las materias primas. Los indicadores económicos ayudaron a Correa y a su organización en aquel momento, MPAIS, a mantener una tasa de aprobación promedio del 50 \% (Meléndez y Moncagatta, 2017; LAPOP, 2016). Correa utilizó la bonanza económica para fortalecer el papel del Estado, creó empresas estatales para controlar varios sectores productivos en Ecuador, como recursos hídricos, telecomunicaciones, energía, minería y recursos naturales no renovables. El tamaño del Estado aumentó drásticamente por la creación de nuevos ministerios - en general, el crecimiento del ejecutivo- y el aumento del gasto social (Jara-Alba y Umpiérrez de Reguero, 2014).

En 2012-2013, la caída de los precios del petróleo y el aumento de la deuda externa, principalmente con China, obstaculizaron una expansión económica fuerte y afectaron la inversión pública y el gasto social (Gallagher, Irwing y Ko- 
leswi, 2013). La economía entró en recesión en 2015, dos años antes del final del tercer mandato de Correa (Vera Rojas y Llanos Escobar, 2016). Correa respondió a la desaceleración económica manteniéndose más fuerte en el poder. Sin embargo, las condiciones económicas perjudicaron paulatinamente el apoyo electoral a Correa y MPAIS. En las elecciones locales y regionales de 2014, MPAIs perdió el $10,8 \%$ de los escaños y varios gobiernos locales estratégicos, incluido casi el $75 \%$ de las capitales de provincia, en comparación con 2009. Los partidos de derecha tradicional no ganaron de manera abrumadora. Dos partidos emergentes, CREO Y SUMA aumentaron su cuota de asientos a expensas de MPAIS.

Esta oportunidad, del lado de la demanda permitió a CREO posicionarse en 2013 como la segunda fuerza política más grande después de MPAIS. CREO se benefició de la imagen descendente de otros partidos y movimientos políticos de derecha, retratada en el ataque continuo de Correa a la partidocracia. Así, cuando Correa logró intimidar al viejo sistema de partidos, CREO pudo llenar el vacío como un partido de derecha emergente. En su ascenso, CREO también aprovechó los incentivos del sistema electoral ecuatoriano.

Desde 1998 hasta 2019 el sistema para elecciones pluripersonales era proporcional de lista abierta con panachage, el electorado podía elegir a más de un candidato de diferentes listas y partidos (Mustillo y Polga-Hecimovich, 2018; Pachano, 2004; Umpiérrez de Reguero y Dandoy, 2021). La reforma electoral de 2019 cambió varios elementos del sistema electoral, reemplazó el panachage por una lista cerrada y bloqueada, aunque con un método de asignación de escaños proporcional para elecciones pluripersonales, lo que en términos generales empujaría a un sistema electoral con partidos ligeramente más fuertes.

En estas últimas décadas, las reglas electorales han proporcionado diferentes mecanismos de conversión de votos en escaños, y una distribución ajustable de los criterios demográficos y geográficos de las circunscripciones electorales en función del tipo de elección (Freidenberg y Pachano, 2016; Nohlen, 2012; Sánchez, 2008). Por ejemplo, en las elecciones legislativas, hubo dos métodos de asignación de escaños, d'Hondt y Webster, para niveles geográficos provinciales y nacionales, respectivamente, de 2012 a 2019. En las últimas elecciones de 2021, todos los votos legislativos se contaron con Webster, aumentando moderadamente la proporcionalidad del voto y la fragmentación partidaria a niveles supranacional, del exterior y provincial. En cualquier caso, esta compleja composición electoral, además de las divisiones sociales que tiene Ecuador como país plurinacional, promueve la creación de múltiples partidos y movimientos políticos, así como de alianzas y coaliciones multipartidistas a corto plazo y puntuales, lo que la literatura denomina como flash parties (Mustillo, 2007, p. 16). De hecho, los partidos — según la ley electoral vigente — pueden formar alianzas 
estratégicas para optimizar su participación en términos de votos y escaños, dado el apoyo electoral esperado. Afirmamos que CREO utilizó con éxito los incentivos de las reglas electorales para posicionarse como el partido de derecha a nivel nacional más fuerte de Ecuador durante la última década.

\subsection{Del lado de la oferta}

De 2013 a 2021 CREO se posicionó como el principal partido de oposición. Después de que el candidato elegido por Correa, Lenín Moreno, asumiera la presidencia en 2017, Moreno y Correa tuvieron una división notoria que finalmente llevó a la ruptura interna de MPAIS (Wolff, 2018). Habiendo ganado principalmente por la popularidad de Correa, Moreno, quien anteriormente se desempeñó como vicepresidente de Correa (2007-2013), se distanció de Correa y perdió el apoyo de alrededor del $38 \%$ de la bancada legislativa de MPAIS, en la cohorte de 2017-2021. En enero de 2018, 28 asambleístas se desafiliaron de MPAIS y formaron el movimiento Revolución Ciudadana (RC) para apoyar a Correa, brindaron, así, las mismas condiciones en la legislatura unicameral que antes de 2009: altos niveles de fragmentación y, por tanto, ningún partido con mayoría absoluta (Acosta, 2009).

El líder de CREO, Guillermo Lasso, quien perdió ante Moreno en 2017, aprovechó la oportunidad y ofreció apoyo de los legisladores de CREO al gobierno si Moreno implementaba reformas favorables al mercado. La disputa de Moreno con Correa llevó al primero a convocar un referéndum a principios de 2018 para restablecer los límites de mandato en todos los cargos de elección directa. Si bien se había instituido un límite de dos mandatos en la Constitución de 2008, esta barrera legal se había eliminado desde 2015 mediante una reforma constitucional diseñada para permitir que Correa se postulara para la reelección —una opción que sorprendentemente se negó a usar en 2017. El referéndum iniciado por Moreno buscó prohibir que Correa se postule nuevamente para presidente en 2021. Lasso y CREO hicieron campaña activamente por el voto del Sí en dicho mecanismo de democracia directa. Aunque CREO y Moreno no se han convertido visiblemente en aliados, CREO ha colaborado con Moreno en la promoción de reformas promercado, una campaña anticorrupción y una reforma constitucional sobre límites de mandato (Labarthe y Saint-Upéry, 2017; Wolff, 2018).

En 2018, los legisladores de CREO jugaron un papel importante en los debates plenarios contra los escándalos de corrupción, específicamente los originados por las negociaciones informales entre políticos de alto perfil y Odebrecht, la constructora brasileña asociada a sobornos y devoluciones de contratos gubernamentales en diferentes países, incluido Ecuador. Entre 2018 y principios de 
2021, partidos y movimientos de derecha, como CREO, SUMA y PSC, se unieron a MPAIS de Moreno, en la campaña gubernamental para combatir la corrupción que el expresidente Correa y sus coidearios alegaron era una caza de brujas destinada a prevenir reinsertar al mismo Correa en la política.

La estructura interna de CREO es flexible, dentro y fuera de Ecuador. Dado que la campaña del partido y las estrategias de comunicación desde su fundación se han centrado en su candidato presidencial, CREO puede verse fácilmente como un vehículo electoral de Guillermo Lasso (Moncagatta y Espinosa, 2019, p. 134). En efecto y similar a Mauricio Macri con Propuesta Republicana (PRO) en Argentina (Vommaro, 2019), Lasso ha sido el primus inter pares de CREO desde su fundación. En gran medida, el diseño, la coordinación y la ejecución de las campañas presidenciales y legislativas han evolucionado en torno a Lasso, tanto en el ámbito nacional como entre los ecuatorianos en el exterior. No obstante, CREO tiene el potencial de convertirse en un caso de construcción partidaria exitosa, si mantiene al menos el $10 \%$ de los votos a nivel nacional y cambia su liderazgo en el futuro; caso contrario, seguirá siendo personalista mientras su desempeño electoral lo permita (Levitsky, Loxton y Van Dyck, 2016).

En el tercer período presidencial de Correa (2013-2017), CREO estructuró un mensaje claro como partido de oposición. Mientras abogaba por el respeto a las libertades individuales y la democracia como la mejor forma de gobierno, CREO criticó lo que varios autores han calificado como el descenso de Correa al autoritarismo competitivo (Basabe-Serrano y Martínez, 2014; Meléndez y Moncagatta, 2017; Levitsky y Loxton, 2013). Por consiguiente, se puede pensar que cREo puede ser más que un partido creado para lograr que Lasso llegue a la presidencia. En 2017-2018, cuando Moreno asumió la presidencia, CREO colaboró de forma selectiva, ya que la administración de Moreno adoptó políticas más moderadas e incluso favorables al mercado. CREO ha mantenido un discurso coherente con el sector privado empresarial y las instituciones conservadoras de la sociedad civil.

\section{Historia e ideología}

CREO es la abreviatura de Movimiento Creando Oportunidades. Esta organización política se registró oficialmente en el Consejo Nacional Electoral (CNE) el 20 de enero de 2012. La identificación en la papeleta electoral de CREO es 21, desde sus primeras elecciones en 2013.

Guillermo Lasso, un hombre de negocios, nacido en 1955, que instituyó el Banco de Guayaquil en la década de 1990, uno de los tres bancos más grandes de Ecuador, fundó CREO. Lasso y varios líderes de CREO están asociados con el 
Opus Dei - Iglesia católica- (Arévalo Gross, 2017) y dos centros de estudios de derecha (Inteligencia Estratégica y Ecuador Libre) relacionados con los valores económicos liberales.

Lasso ingresó a la política como gobernador de la provincia de Guayas y superministro de economía bajo el gobierno de Jamil Mahuad (1998-1999). Presidió los primeros meses de la crisis bancaria de 1999-2000 que llevó a la adopción del dólar estadounidense como moneda nacional. Sus críticos alegan que favoreció a su propio banco en detrimento de otros que quebraron durante la crisis (Jara-Alba y López-Guzmán, 2015). Bajo Lasso, el Banco de Guayaquil implementó una opción bancaria de bajo costo. El Banco del Barrio tiene presencia en todo el país y es popular en los sectores rurales y de bajos ingresos. En los últimos años, Lasso pasó la propiedad del Banco de Guayaquil a sus hijos.

Similar a sistemas partidarios donde la derecha está dividida ideológicamente como en Perú (Meléndez, 2019), la derecha ecuatoriana es diversa en términos de institucionalización, estilo de liderazgo y posiciones ideológicas (Moncagatta y Espinosa, 2019). En este marco, CREO aboga por una mezcla de ideas liberales y conservadoras. Es liberal desde la perspectiva económica, así como conservador en cuestiones sociales y morales (Herrera et al., en edición). Esta organización apoya los valores provida, el respeto a los derechos humanos y defiende la democracia como la mejor forma de gobierno. En su declaración de principios, CREO comparte la normativa establecida por la Constitución de Montecristi (2008) sobre la democracia representativa, directa y participativa. Sin embargo, su conceptualización de la democracia se adhiere a la alternancia de poder como condición necesaria para su buen funcionamiento.

Las opiniones económicas de CREO pueden ubicarse en el extenso dominio teórico del liberalismo económico. CREO apoya la promoción de una economía libre, el emprendimiento y la igualdad de oportunidades laborales (Herrera et al., en edición). Esta organización política oscila entre el ordoliberalismo promovido por la Escuela de Economía de Friburgo y los axiomas clásicos de la Escuela de Economía de Austria.

Al competir en elecciones presidenciales y de otro tipo, CREO ha adoptado diversas estrategias para atraer votantes al interior del Ecuador y en las circunscripciones especiales del exterior. Para las elecciones nacionales de 2013, CREO compitió como un partido político independiente con presencia a nivel nacional. Para los escaños de la Asamblea Nacional a nivel provincial, CREO se unió a coaliciones multipartidistas, con partidos de derecha en algunas provincias y organizaciones políticas personalistas o con intereses únicamente locales en otras. Por ejemplo, CREO formó una alianza con sUMA y Alianza Tsáchila en la provincia de 
Santo Domingo de los Tsáchilas, así como una coalición diferente con el partido tradicional de derecha, PSC, en la provincia de Los Ríos.

En las elecciones regionales y locales de 2014, CREO volvió a formar diferentes coaliciones preelectorales dependiendo de la estructura de oportunidades en las diferentes unidades electorales. En varios distritos, CREO unió fuerzas con organizaciones políticas regionales, locales o nacionales para optimizar su porcentaje de votos y ganar más escaños. Para las elecciones de la prefectura en la provincia de Los Ríos, CREO se alió con varios grupos de oposición — PSC, Partido Sociedad Patriótica (PSP), Partido Renovador Institucional Acción Nacional (PRIAN), Partido Roldosista Ecuatoriano (PRE) y SUMA - aun así, perdió contra MPAIS. A nivel municipal, en 2014, CREO formó principalmente alianzas con organizaciones políticas locales y regionales, como el Movimiento Independiente Libertad en la provincia de Santa Elena o Alianza Democracia Radical en la provincia de Morona Santiago.

Aunque la constitución de 2008 fue hecha a la medida de Correa (Basabe-Serrano y Martínez, 2014; Montúfar, 2016; Pachano, 2012), incluyó un límite de dos mandatos para los cargos electos para la presidencia. Después de que Correa fuese elegido en 2013 para su segundo mandato bajo el nuevo marco jurídico, algunos de sus partidarios buscaron abolir los límites de mandato. CREO lideró la oposición de cara a la abolición de los límites de mandato. Se convirtió en el principal oponente al esfuerzo del presidente Correa de permanecer en el cargo por tiempo indefinido.

CREO presentó dos Amicus Curiae ante la Corte Constitucional de Ecuador e hizo campaña a favor de una iniciativa de referéndum sobre límites de mandatos. Las diferentes instituciones públicas nacionales del Ecuador negaron todas estas acciones legales y se promulgó la reforma constitucional. Sorprendentemente, Correa - después de ser declarado elegible para postularse para un nuevo mandato- negó su repostulación, porque quería quedarse fuera por un mandato y regresar en 2021.

La tabla 1 muestra el cronograma del proyecto de enmiendas constitucionales que contiene la reelección indefinida (2014-2015) apoyada por los coidearios de Correa y con la oposición activa de CREO. Luego de que los coidearios de Correa impulsaran con éxito este proyecto de enmiendas a través de la Corte Constitucional, CREO realizó un esfuerzo para iniciar un referéndum sobre el tema. Finalmente, la Corte Constitucional negó la solicitud para que se realizara un referéndum sobre la abolición de los límites de mandato y se promulgó el conjunto de enmiendas constitucionales que abolió los límites de mandato. La reelección indefinida se aplicó a todos los cargos electos. 


\section{Tabla 1. Cronología del proyecto de enmiendas constitucionales para la reelección indefinida (2014-2015)}

\begin{tabular}{|c|c|}
\hline Fecha & Actividad \\
\hline 22 de mayo de 2014 & $\begin{array}{l}\text { Soledad Buendía, para aquel entonces asambleísta de } \\
\text { MPAIs, presentó el proyecto de enmiendas constitucionales } \\
\text { en la Asamblea Nacional, que contenía la iniciativa de } \\
\text { reelección indefinida. }\end{array}$ \\
\hline 26 de junio de 2014 & $\begin{array}{l}\text { Siguiendo el proceso constitucional, la entonces presiden- } \\
\text { ta de la Asamblea Nacional, Gabriela Rivadeneira, facilitó } \\
\text { la propuesta de enmiendas para la revisión de la Corte } \\
\text { Constitucional. }\end{array}$ \\
\hline 31 de junio de 2014 & $\begin{array}{l}\text { La Corte Constitucional admitió revisar la causa (\#0001- } \\
14-\mathrm{RC} \text { ) sobre el proyecto de enmiendas constitucionales. }\end{array}$ \\
\hline 21 de julio de 2014 & $\begin{array}{l}\text { Guillermo Lasso, líder de CREO, a través de un Amicus } \\
\text { Curiae, pidió a la Corte Constitucional rechazar este } \\
\text { proyecto de enmiendas por razón de inconstitucionalidad. }\end{array}$ \\
\hline 12 de agosto de 2014 & $\begin{array}{l}\text { Wendy Molina Andrade fue nombrada la jueza a cargo de } \\
\text { revisar la causa (\#0001-14-RC). }\end{array}$ \\
\hline 31 de octubre de 2014 & $\begin{array}{l}\text { La Corte Constitucional promulgó un dictamen favorable } \\
\text { a la reelección indefinida. }\end{array}$ \\
\hline $\begin{array}{l}10 \text { de noviembre de } \\
2014\end{array}$ & $\begin{array}{l}\text { El veredicto de la Corte Constitucional fue publicado en } \\
\text { la Gaceta Oficial n. }{ }^{\circ} 009 .\end{array}$ \\
\hline 11 de febrero de 2015 & $\begin{array}{l}\text { CREO sometió a consideración una iniciativa en el CNE } \\
\text { para celebrar una consulta popular sobre la reelección } \\
\text { indefinida. }\end{array}$ \\
\hline 13 de febrero de 2015 & $\begin{array}{l}\text { El CNE negó la propuesta de CREO sobre el mecanismo de } \\
\text { democracia directa en torno a la reelección indefinida. }\end{array}$ \\
\hline 12 de marzo de 2015 & $\begin{array}{l}\text { CREO introdujo un segundo Amicus Curiae ante la Corte } \\
\text { Constitucional requiriendo un dictamen sobre los proce- } \\
\text { dimientos para llevar a cabo una iniciativa popular acerca } \\
\text { de la reelección indefinida. La Corte Constitucional negó } \\
\text { esta petición. }\end{array}$ \\
\hline $\begin{array}{l}3 \text { de diciembre de } \\
2015\end{array}$ & $\begin{array}{l}\text { La Asamblea Nacional aprobó el proyecto de enmiendas } \\
\text { constitucionales con } 100 \text { votos a favor, } 8 \text { en contra y } 1 \\
\text { abstención. Los asambleístas de CREO no estuvieron pre- } \\
\text { sentes en la sala del pleno de la Asamblea Nacional para } \\
\text { la votación. }\end{array}$ \\
\hline
\end{tabular}

Fuente: Umpiérrez de Reguero (2016, pp. 222-223). 
En 2016, antes de la campaña electoral presidencial de 2017, CREO se alió con el PSC. Pero cuando el PSC nominó a Cynthia Viteri como su candidata presidencial, PSC y CREO se convirtieron en competidores. La colaboración preelectoral con otras organizaciones de oposición a la presidencia de Correa que había defendido CREO resultó en una alianza con SUMA a nivel nacional. Esta coalición preelectoral participó como una sola fuerza en todos los procesos electorales de 2017, incluidas las elecciones andinas (Dandoy, 2017).

En las elecciones autonómicas y locales de 2019 CREO volvió a formar distintas coaliciones preelectorales, muchas de ellas con más de una organización política. En la provincia de Loja, CREO ganó su única sede en la prefectura junto con dos partidos nacionales (Adelante Ecuador Adelante [AEA, ex PRIAN] y Fuerza Ecuador [FE, ex PRE]) y tres movimientos locales (Juntos Podemos, Convocatoria y Unión Ecuador). A nivel municipal, CREO fue la segunda fuerza electoral de derecha después del PSC, inclusive antes del partido incumbente, MPAIS.

\section{Resultados electorales por tipo de elecciones}

En la votación de la primera vuelta de las elecciones presidenciales de 2013, Lasso - el candidato presidencial de CREO- ocupó el segundo lugar detrás de Rafael Correa. Correa ganó la presidencia sin balotaje. En la legislatura, Mae Montaño, en ese entonces jefa de la bancada de CREO para las elecciones legislativas, y otros nueve candidatos legislativos fueron elegidos como miembros de la Asamblea Nacional.

Desde 2014, CREO formó varios grupos políticos (por ejemplo, Compromiso Ecuador) y apoyó a algunos candidatos de derecha. En las elecciones regionales y locales de 2014, CREO obtuvo 22 alcaldías, incluidas tres en alianza con otras organizaciones políticas locales y regionales de derecha, así como una de las 23 prefecturas correspondiente a la provincia de Loja, en la región Sierra sur del país.

Para las elecciones presidenciales de 2017, CREO formó la coalición Unidos por el Cambio como plataforma electoral y política de Lasso. En las elecciones legislativas de 2017, la alianza con suma obtuvo 34 escaños en la Asamblea Nacional, convirtiéndose en el bloque de oposición más importante en la administración de Moreno.

Lasso se postuló como candidato presidencial de CREO en 2017 por segunda vez; en esta ocasión, llegó al balotaje. En la segunda vuelta, Moreno (de MPAIS) ganó por un margen de 2,3 \%, la victoria más estrecha desde 1998. 
CREO incrementó su desempeño electoral en las elecciones locales de 2019. Esta organización política obtuvo un aumento del $50 \%$ en términos de escaños: de 22 alcaldías en 2014 a 33 alcaldías en 2019. Por su parte, en la elección de prefectura de 2019, tanto CREO como otras organizaciones políticas (PSP, ID y SUMA) mantuvieron sus posiciones en comparación a las elecciones seccionales de 2014. En estas elecciones locales y regionales su alianza con SUMA ya no fue tan fuerte como en elecciones anteriores. De hecho, solo ganaron una alcaldía en la provincia de Guayas al participar juntos. Desde mediados de 2018 CREO y SUMA se distanciaron como consecuencia, presuntamente, de su respectivo comportamiento electoral en el pleno de la Asamblea Nacional. En general, ambos partidos presentaron diferentes preferencias en repetidas ocasiones. A finales de 2018, SUMA se convirtió en un aliado importante del PSC.

En 2021, la coalición entre CREO y el PSC postuló a Lasso como candidato presidencial por tercera vez consecutiva. Guillermo Lasso casi no entró a la segunda vuelta. Por menos de medio punto porcentual (lo que equivale a 32.115 votos), Yaku Pérez (Pachakutik) estuvo cerca de arrebatarle su posibilidad de continuar en la papeleta frente a Andrés Arauz (Unión por la Esperanza [UNES] $]^{4}$ ). En el balotaje, Lasso venció a Arauz con una diferencia de casi $5 \%$ —alrededor de 425.000 votos - , lo que significa la primera victoria en una elección directa de un candidato abiertamente de derecha desde la década de 1990.

En la Asamblea Nacional, sin embargo, CREO tuvo un severo declive, en parte por el cambio de método de asignación de escaños en elecciones pluripersonales a nivel provincial. En referencia a las elecciones legislativas de 2017, CREO obtuvo 22 escaños menos $(64,71 \%$ menos que el número de escaños totales obtenidos anteriormente). La tabla 2 resume la participación promedio de votos de CREO por tipo de elecciones desde 2013 hasta las últimas elecciones de 2021.

4 UNES es la coalición procorreísta, entre las organizaciones políticas, Revolución Ciudadana y Centro Democrático. 


\section{Tabla 2. Promedio de votos de creo por tipo de elección,} en porcentajes (2013-2021)

\begin{tabular}{lcccc} 
& $2013-2014$ & $2017-2019$ & 2021 & Promedio \\
\hline $\begin{array}{l}\text { Elecciones suprarre- } \\
\text { gionales }\end{array}$ & 11,42 & 18,92 & 8,58 & 16,19 \\
$\begin{array}{l}\text { (Parlamento Andino) } \\
\begin{array}{l}\text { Elecciones } \\
\text { presidenciales } \\
\text { (primera vuelta) }\end{array}\end{array}$ & 22,68 & 28,09 & 19,74 & 23,50 \\
$\begin{array}{l}\text { Elecciones } \\
\text { presidenciales } \\
\text { (segunda vuelta) }\end{array}$ & - & 48,84 & 52,47 & 50,66 \\
$\begin{array}{l}\text { Elecciones } \\
\text { legislativas } \\
\text { (asientos nacionales) }\end{array}$ & 11,39 & 20,10 & 9,65 & 13,71 \\
$\begin{array}{l}\text { Elecciones } \\
\text { legislativas } \\
\text { (asientos provinciales) }\end{array}$ & 11,25 & 20,09 & 14,58 & 15,31 \\
$\begin{array}{l}\text { Electorales regionales } \\
\text { (prefectura) }\end{array}$ & 11,50 & 9,57 & & 10,54 \\
$\begin{array}{l}\text { Elecciones locales } \\
\text { (alcaldías) }\end{array}$ & 12,36 & 9,93 & - & \\
\hline
\end{tabular}

Nota: En 2013, 2017 y 2021 se realizaron elecciones andinas, presidenciales y legislativas a nivel nacional. En 2014 y 2019, se realizaron elecciones locales (alcaldias) y regionales (prefecturas). Fuente: CNE (2021).

\section{Resultados electorales por provincia}

CREO ha tenido una presencia relativamente fuerte a nivel nacional, especialmente en las provincias del centro, sur y este de Ecuador como Chimborazo, Loja y Zamora Chinchipe. No obstante, en términos de tamaño poblacional, las provincias en las que CREO ha tenido un porcentaje medio y alto de votos son de menor importancia, en comparación con provincias demográficamente relevantes como Guayas y Manabí, como se muestra en la figura 1. A pesar de estar asociado a la región costera de Ecuador, particularmente a Guayaquil, donde Lasso hizo carrera como banquero y obtuvo su primer cargo político, CREO ha desafiado la clásica división regional que ha caracterizado a la política ecuatoriana. La división regional ha dividido el sistema partidario ecuatoriano en regiones geográfi- 
cas, en particular Costa y Sierra, en donde las fuerzas políticas normalmente han sido más fuertes en una $u$ otra. Los partidos nacionales han tenido un discurso nacional, pero a menudo una presencia regional profundamente arraigada. Según Freidenberg y Alcántara-Sáez (2001), la Costa se asocia a partidos más liberales y populistas; mientras que, la sierra a opciones más tradicionales o conservadoras. Al ganar más votos en las provincias de la Amazonía y la sierra ecuatoriana, a CREO se lo puede describir con seguridad como un partido de apelación nacional.

\section{Figura 1. Proporción de voto por CREO en elecciones presidenciales por provincia}

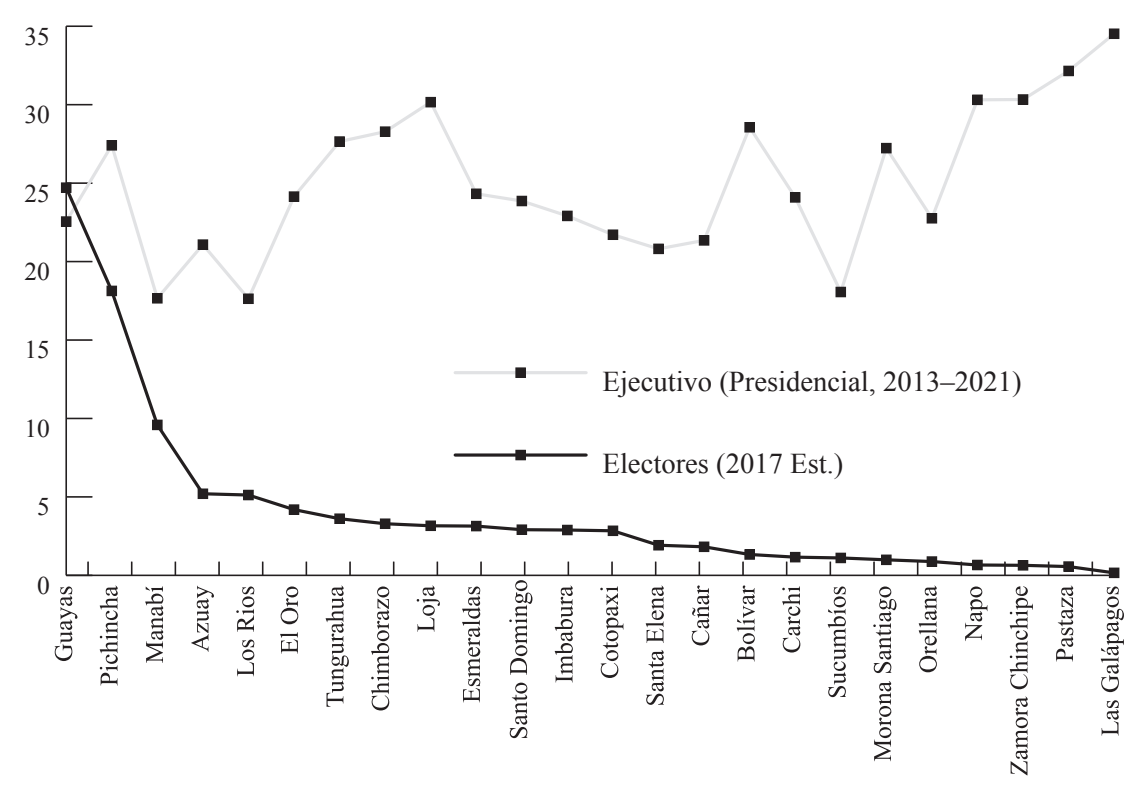

Fuente: CNE (2021).

A nivel electoral local y regional, se puede observar el mismo patrón. En Galápagos, Pastaza, Zamora Chinchipe y Loja, por ejemplo, CREO ha obtenido excelentes resultados dado el sistema político ecuatoriano altamente fragmentado (Freidenberg, 2012; Pachano, 2010). En las elecciones locales y regionales, donde el sistema electoral ofrece fuertes incentivos para crear organizaciones políticas y formar coaliciones electorales en todos los niveles electorales y geográficos, incluidos los distritos en el extranjero, ganar más del $25 \%$ de los votos en varios distritos es un logro importante (Dandoy, 2014; Freidenberg, 2006; Freidenberg y Pachano, 2016; Ortiz, 2012; Umpiérrez de Reguero y Dandoy, 2021). 
64 CREO: el ascenso y los desafíos de consolidación del partido político de derecha emergente en Ecuador

Aun así, pese a una buena actuación, hay provincias enteras, como Manabí y Carchi en las que CREO no obtuvo una sola alcaldía o representación de prefectura.

La tabla 3 resume la participación promedio de votos CREO por provincia de 2013 a 2019. La quinta columna, Ejecutivo (GAD o gobierno autónomo descentralizado) considera el número de escaños para obtener la participación promedio. También muestra el porcentaje de habitantes registrados para votar (estimado en 2017). Hemos incluído esta columna para medir los diferentes pesos relativos a cada provincia, ya que Guayas, Manabí y Pichincha han comprendido no solo una distribución electoral distinta, sino un determinante significativo para el éxito electoral y la ambición política (Basabe-Serrano y Martínez, 2014; Polga-Hecimovich, 2013). De hecho, es gracias al voto de Pichincha que Lasso obtuvo una ventaja relevante en el balotaje de 2021 frente a Arauz, además presumiblemente del alto número de votos nulos y blancos (alrededor de 17\%). 
Tabla 3. Promedio de cuotas de voto de CREO por provincia (2013-2021)

\begin{tabular}{lccccc} 
& Electores* & $\begin{array}{c}\text { Ejecutivo } \\
\text { (presidencial) }\end{array}$ & $\begin{array}{c}\text { Legislativo } \\
\text { (asientos } \\
\text { nacionales) }\end{array}$ & $\begin{array}{c}\text { Ejecutivo } \\
\text { (GAD**) }\end{array}$ & $\begin{array}{c}\text { Legislativo } \\
\text { (asientos } \\
\text { provinciales) }\end{array}$ \\
\hline Guayas & 24,71 & 22,55 & 9,73 & 6,49 & 7,47 \\
Pichincha & 18,13 & 27,41 & 18,35 & 11,69 & 13,23 \\
Manabí & 9,59 & 17,66 & 10,38 & 8,54 & 11,30 \\
Azuay & 5,20 & 21,08 & 15,05 & 9,04 & 13,78 \\
Los Rios & 5,12 & 17,63 & 9,41 & 18,49 & 11,15 \\
El Oro & 4,19 & 24,14 & 11,10 & 10,02 & 6,22 \\
Tungurahua & 3,61 & 27,65 & 17,14 & 7,03 & 17,43 \\
Chimborazo & 3,29 & 28,28 & 17,97 & 16,36 & 13,33 \\
Loja & 3,16 & 30,16 & 21,64 & 26,74 & 21,64 \\
Esmeraldas & 3,14 & 24,32 & 15,26 & 1,83 & 14,18 \\
Santo Domingo & 2,91 & 23,86 & 16,65 & 7,73 & 16,04 \\
de los Tsáchilas & & 22,91 & 14,52 & 5,23 & 12,43 \\
Imbabura & 2,89 & 21,71 & 12,79 & 8,86 & 12,30 \\
Cotopaxi & 2,84 & 20,81 & 14,13 & 10,29 & 9,54 \\
Santa Elena & 1,92 & 21,35 & 15,20 & 24,04 & 17,36 \\
Cañar & 1,82 & 28,56 & 17,87 & 11,40 & 11,68 \\
Bolívar & 1,33 & 24,09 & 13,57 & 1,71 & 7,67 \\
Carchi & 1,16 & 18,06 & 10,26 & 4,77 & 9,30 \\
Sucumbíos & 1,11 & 27,23 & 16,36 & 18,65 & 19,26 \\
Morona & 0,99 & & & & \\
Santiago & & 22,76 & 12,95 & 19,43 & 10,61 \\
Orellana & 0,88 & 30,31 & 12,53 & 10,25 & 8,53 \\
Napo & 0,66 & 15,95 & 8,23 & 14,03 \\
Zamora & 0,64 & 30,33 & & 5,12 & 16,50 \\
Chinchipe & & 32,15 & 20,28 & 5,96 \\
Pastaza & 0,56 & 34,52 & 18,78 & 6,05 & 19,96 \\
Las Galápagos & 0,16 & & & &
\end{tabular}

Notas: (*) Estimaciones de 2017. (**) GAD es la abreviatura de Gobiernos Autónomos Descentralizados, que comprenden el gobierno regional y local en Ecuador.

Fuente: CNE (2021). 


\section{Resultados electorales en el extranjero}

Existen al menos dos tipos de sistemas para que los nacionales no-residentes voten desde el extranjero: 1. votar en el extranjero por el distrito de origen, y 2. votar en el extranjero para la representación directa (Collyer, 2014). Desde 2007, Ecuador ha pasado de implementar el primer tipo al segundo tipo de votación desde el exterior (Umpiérrez de Reguero y Dandoy, 2020). El sistema ecuatoriano de votación en el exterior para la representación directa permite a los emigrantes elegir seis legisladores en la Asamblea Nacional en tres distritos binominales: Canadá y Estados Unidos; América Latina, el Caribe y África; Europa y otros lugares. De hecho, en comparación con la minoría de países que brindan una representación especial de emigrantes, Ecuador es el único caso que sobrerrepresenta a sus nacionales residentes en el exterior, en relación con la representación de sus ciudadanos en provincias dentro del país (Collyer, 2014) o que extiende una representación casi proporcional a sus emigrantes (Palop-García, 2018). El ejemplo más claro es la circunscripción de América Latina, el Caribe y África, que tiene menos de 100.000 ecuatorianos inscritos para votar, pero aún tiene dos escaños en la legislatura asignados por el CNE.

La disposición del voto en el exterior en Ecuador no solo tiene el incentivo adicional de esta representación, sino que también permite a los nacionales no residentes votar en seis tipos de elecciones, la mayoría de las veces simultáneamente (Umpiérrez de Reguero y Dandoy, 2020), lo que es una anomalía en términos de provisión legal en comparación a la región (Arrighi et al., 2019). Los ecuatorianos residentes en el exterior pueden votar en las elecciones para elegir un binomio presidencial, asambleístas nacionales, legisladores emigrantes, parlamentarios andinos, miembros del Consejo de Participación Ciudadana y Control Social (CPCCS), así como emitir sus preferencias en referendos y consultas populares nacionales. En suma, la provisión vigente que brinda Ecuador a sus nacionales no-residentes es una de las más generosas de la región.

El voto de los nacionales no-residentes históricamente ha favorecido a MPAIS debido a, primero, las características del gran flujo de emigración ecuatoriana asociado a la crisis económica de 1999-2000 denominado Feriado Bancario (Boccagni y Ramírez, 2013; Jara-Alba y López-Gúzman, 2015); y segundo, la entrada estratégica de MPAIs para captar votos en el exterior (Umpiérrez de Reguero y Dandoy, 2021) — enarbolada por la referencia de Correa a la Quinta Región en su discurso (Ramírez, 2018). ${ }^{5}$ Ahora bien, en las elecciones generales de

5 La Quinta Región es una construcción metageográfica que busca incorporar a los nacionales residentes en el exterior como una región más del país (aparte de Amazonía, Costa, Galápagos y Sierra). 
2017, hubo un cambio en el comportamiento electoral de los ecuatorianos no-residentes. CREO ganó dos de los seis escaños que anteriormente ocupaba MPAIs: uno por la circunscripción de Estados Unidos y Canadá, y otro por la circunscripción de América Latina, el Caribe y África. De hecho, Lasso ganó en estas dos circunscripciones en la segunda vuelta de las elecciones presidenciales de 2017. Empero, Lenín Moreno ganó la presidencia por el total de votos en el exterior, ya que la circunscripción de Europa, Asia y Oceanía tiene mayor peso electoral en comparación con los dos distritos antes indicados (CNE, 2021).

En el 2021, CREO solo mantuvo su asiento en la circunscripción de América Latina, El Caribe y África, mientras que Pachakutik le arrebató el de Estados Unidos y Canadá. De manera similar a 2017, Lasso pierde en el exterior por la circunscripción de Europa, Asia y Oceanía frente a Arauz. La tabla 4 describe las acciones de voto promedio de CREO de 2013 a 2021.

Tabla 4. Proporción de voto para CREO en el exterior (2013-2021)

\begin{tabular}{lccc} 
& Exterior & Interior & Diferencia \\
\hline $\begin{array}{l}\text { Elecciones suprarregionales } \\
\text { (Parlamento Andino) }\end{array}$ & 14,89 & 14,05 & 0,84 \\
$\begin{array}{l}\text { Elecciones presidenciales } \\
\text { (primera vuelta) }\end{array}$ & 19,01 & 22,53 & $-3,52$ \\
$\begin{array}{l}\text { Elecciones presidenciales } \\
\text { (segunda vuelta) }\end{array}$ & 48,68 & 50,61 & $-1,93$ \\
$\begin{array}{l}\text { Elecciones legislativas } \\
\text { (asientos nacionales) }\end{array}$ & 13,20 & 13,50 & $-0,30$ \\
$\begin{array}{l}\text { Elecciones legislativas } \\
\text { (asientos provinciales) }\end{array}$ & 16,00 & 13,90 & 2,10 \\
$\begin{array}{l}\text { Proporción de cReo del total } \\
\text { del electorado nacional* }\end{array}$ & 1,40 & 98,60 & $-97,20$ \\
$\begin{array}{l}\text { Proporción del total } \\
\text { del electorado nacional* }\end{array}$ & 2,98 & 97,02 & - \\
\hline
\end{tabular}

Nota: (*) Estimaciones de 2017.

Fuente: CNE (2021)

En general, la participación de votantes en las circunscripciones del exterior es a menudo menor que la participación a nivel nacional. Con alrededor del $10 \%$ de los ecuatorianos viviendo en el exterior, la participación en las circunscripciones del exterior oscila entre $30 \%$ y $40 \%$, lo que hace que valga la pena discutir 
el voto de los ecuatorianos no residentes. En las elecciones de 2017, la movilización electoral de nacionales no-residentes fue comparable a la de provincias de mediana escala de Ecuador; de hecho, muy similar a Bolívar o Cañar en votos, y a Cotopaxi en número de habitantes registrados en el proceso electoral (tabla 5).

Tabla 5. Comparación de votos por provincias de mediana escala y en el exterior (2017 elección)

\begin{tabular}{lcccc} 
Provincia & $\begin{array}{c}\text { Votantes } \\
\text { habilitados }\end{array}$ & $\begin{array}{c}\text { Porcentaje a } \\
\text { nivel nacional }\end{array}$ & Votantes & $\begin{array}{c}\text { Porcentaje a } \\
\text { nivel nacional }\end{array}$ \\
\hline Exterior & 378.251 & 2,95 & 135.867 & 1,44 \\
Cotopaxi & 353.357 & 2,76 & 288.608 & 3,06 \\
Santa Elena & 239.197 & 1,87 & 208.505 & 2,21 \\
Bolívar & 165.366 & 1,29 & 131.214 & 1,39 \\
Cañar & 225.783 & 1,76 & 140.629 & 1,49 \\
Carchi & 143.914 & 1,12 & 117.832 & 1,25 \\
Ecuador & 12.816 .698 & 100 & 9.442 .495 & 100 \\
(Total) & & & &
\end{tabular}

Fuente: CNE (2017)

\section{Desempeño electoral de los candidatos CREO por género}

Durante la última década, la participación y representación femenina ha aumentado en todas las instituciones políticas ecuatorianas (World Economic Forum, 2016); por ejemplo, en los gabinetes ministeriales (Sotomayor Valarezo y Huertas-Hernández, 2021). En gran medida, las cuotas de género han contribuido a producir este resultado (Castellanos y Ulloa, 2016). Las cuotas se incluyeron para equiparar la representación de las mujeres en la vida pública y política. Ecuador inició en febrero de 1997 con la adopción e implementación de la ley del trabajo (Ley Orgánica de Amparo Laboral de Mujeres, 1997). Sin embargo, no fue hasta la Ley Electoral (2000), actualmente derogada, que se estableció una representación del $30 \%$ en las listas de candidatos a elecciones pluripersonales, siguiendo los lineamientos de la Plataforma de Beijing (1995). El artículo 58 de esta normativa indicaba que el $30 \%$ se incrementaría gradualmente hasta el $50 \%$. Posteriormente, la Ley de Elecciones (2000) se refirió a los principios de secuencialidad y alternancia entre las listas (es decir, en el caso de titulares y representantes suplentes). Finalmente, se consolidó en la actual Ley Orgánica Electoral y de Organizaciones 
Políticas: Código de Democracia (2009, art. 94), la obligatoriedad de la paridad, alternancia y secuencialidad en la configuración de las listas electorales, de cada partido y movimiento político, inclusive alianzas electorales (Umpiérrez de Reguero, Jara-Alba y Cassis Jurado, 2016). En la reforma electoral de 2019, las iniciativas para reducir la brecha de género en términos de participación y representación política continúan avanzando mediante la creación de una cuota obligatoria en las elecciones presidenciales de 2021 en adelante.

A pesar de estos avances, CREO se ha quedado atrás en términos de elección de mujeres. Las candidatas de CREO no tienen tanto éxito como los candidatos de este mismo partido. La tabla 6 muestra que, en tres tipos de elecciones, las mujeres de CREO ocupan cero escaños. Este partido ganó un escaño en las elecciones andinas y fue ocupado por un hombre, tanto en 2013 como en 2017. Lo mismo sucedió en las elecciones de prefectura de 2014. CREO solo obtuvo uno de los 23 escaños existentes, y el candidato electo fue un hombre. Un resultado similar emerge a nivel de alcaldías. No obstante, en la legislatura a nivel nacional y provincial, hubo varias excepciones, particularmente en la cohorte de 2017. En 2013, solo Mae Montaño fue elegida en representación de CREO. En 2017, siete candidatas fueron elegidas en representación de CREO, o de la alianza CREO-SUMA, incluyéndose la reelección de Montaño. En 2021, este número decreció en relación directa a su pérdida de asientos legislativos a nivel provincial. Parece que tanto los votantes de CREO como los candidatos que estos votantes prefieren, son más hombres que mujeres. De hecho, esto quedó en evidencia en las elecciones de 2021, cuando Lasso tuvo que aproximarse al voto femenino en segunda vuelta para ganar la elección. Los votantes de CREO, como una parte considerable de sus líderes, tienden a ser elitistas, antipopulistas, católicos, así como de clase socioeconómica media y alta (Herrera et al., en edición; Moncagatta y Espinosa, 2019). 
Tabla 6. Promedio de asientos de creo por género, en porcentaje (2013-2021)

\begin{tabular}{lccc} 
& $\begin{array}{c}\text { Asientos de } \\
\text { mujeres }\end{array}$ & $\begin{array}{c}\text { Asientos } \\
\text { de CREO }\end{array}$ & $\begin{array}{c}\text { Asientos } \\
\text { de mujer } \\
\text { afiliadas a CREO }\end{array}$ \\
\hline $\begin{array}{l}\text { Elecciones suprarregionales } \\
\text { (Parlamento Andino) }\end{array}$ & 53,33 & 13,33 & 0,00 \\
$\begin{array}{l}\text { Elecciones legislativas } \\
\text { (asientos nacionales) }\end{array}$ & 51,11 & 15,56 & 8,89 \\
$\begin{array}{l}\text { Elecciones legislativas } \\
\text { (asientos provinciales) }\end{array}$ & 40,16 & 13,66 & 3,55 \\
$\begin{array}{l}\text { Electorales regionales } \\
\text { (prefectura) }\end{array}$ & 13,04 & 4,35 & 0,00 \\
$\begin{array}{l}\text { Elecciones locales } \\
\text { (alcaldías) }\end{array}$ & 4,52 & 9,95 & 0,00 \\
\hline
\end{tabular}

Fuente: CNE (2021)

\section{Conclusión}

En 2013, se fundó CREO como respuesta a Rafael Correa y MPAIS. Esta organización política aprovechó las reglas electorales de representación proporcional y llenó en parte el vacío dejado por la desprestigiada imagen de los partidos tradicionales de derecha. La construcción de CREO ha resultado de un desarrollo político idiosincrásico: el ascenso en el poder de Correa y su esfuerzo por cambiar la constitución para permitir la reelección indefinida, así como el creciente anticorreísmo en el electorado. Este análisis proviene de análisis de la oferta y la demanda de CREO.

CREO ha demostrado ser un partido de carácter personalista, pero con presencia nacional, lo que no es tan usual en la política ecuatoriana desde el retorno a la democracia en 1979-1980. Estas características, sumado a la hipotética alternancia de liderazgo en las próximas elecciones presidenciales, podría llevar al partido a ser un caso de construcción partidaria exitosa. Por el momento y a la luz de la literatura (Levitsky, Loxton y Van Dyck, 2016; Mustillo, 2007; Vommaro, 2019), a CREO se lo podría clasificar como un caso de construcción partidaria incompleta, con un componente personalista, focalizado en la candidatura de Lasso. Si bien el candidato presidencial en tres períodos consecutivos (20132021), Guillermo Lasso, no es carismático; en las últimas elecciones nacionales 
obtuvo la presidencia con un holgado porcentaje de diferencia, frente al candidato correísta, Andrés Arauz. Desde su fundación, el desempeño electoral de CREO ha contradicho la histórica división regional entre Costa y Sierra, así como ha contribuido a fortalecer el sistema de partidos frente al proceso de provincialización tradicional (ver Dandoy, 2017 para más detalles sobre esta tendencia del sistema partidario ecuatoriano).

Si bien la derecha en Ecuador confirma la tesis de la resiliencia desarrollada por Luna y Rovira Kaltwasser (2014), a menudo las organizaciones políticas de este espectro ideológico han tenido una fuerte incidencia y liderazgo regional. Moncagatta y Espinosa (2019) argumentan similares conclusiones al poner los ejemplos del PSC y el PRE. En el caso de CREO, hay evidencia que hace pensar lo contrario. Busca ser un partido nacional en todos los niveles de elección. Ello marca la diferencia con otras organizaciones políticas de derecha actuales. Más allá de la figura reconocible de Lasso, CREO se ha posicionado como una de las principales fuerzas electorales opositoras al populismo radical de izquierda de Correa y sus coidearios. En efecto, se podría aseverar que es, en gran parte, gracias al anticorreísmo, que CREO llegó a la presidencia en 2021. Entre 2017 y principios de 2021, CREO ha colaborado con la administración de Lenín Moreno, sin convertirse en un aliado permanente, en la promoción de reformas favorables al mercado, una campaña anticorrupción y una reforma constitucional sobre límites de mandato.

La forma en que CREO ha evolucionado, puede representar una señal de institucionalización en un sistema de partidos tradicionalmente fragmentado como el ecuatoriano y que, desde su origen, ha contrastado con la forma en que MPAIS, luego RC, ha evolucionado. Aunque ambas organizaciones políticas tienen actualmente presencia a nivel nacional, CREO ha surgido y evolucionado desde la sombra de MPAIS/RC para convertirse en un serio competidor electoral nacional desde 2017 y obtener el poder ejecutivo en 2021. Si medimos la institucionalización por los años de sobrevivencia partidaria, la experiencia en elecciones, en un contexto de debilidad institucional (Carreras, 2012; Dávila y Wylie, 2021), entonces CREO tiene buenos prospectos.

Dado que el ascenso electoral de CREO ha estado estrechamente vinculado al desempeño de Guillermo Lasso en las elecciones presidenciales, el partido tiene tanto un desafío como una oportunidad en próximas elecciones. El desafío radica en diversificar sus líderes en futuras elecciones, tanto nacionales como locales y regionales. La oportunidad se estructura a partir de la posible utilización de esa base electoral, de elecciones pasadas, para generar apoyo para el partido más allá de las candidaturas de Lasso (2013-2021) y su reciente administración. 


\section{Referencias}

Acosta, A. M. (2009). Informal coalitions and policymaking in Latin America: Ecuador in comparative perspective. London, United Kingdom: Routledge.

Arévalo Gross, L. (30 de marzo de 2017). El salvador. GK. Recuperado de: https:// elecciones2017.gk.city/2017/03/30/quien-es-guillermo-lasso-candidato-decreo/

Arrighi, J. T., R. Bauböck, D. Hutcheson, A. Ostling, L. Piccoli (2019). Explanatory note: Conditions for Electoral Rights 2019, San Domenico di Fiesole, Italy: European University Institute.

Asamblea Nacional del Ecuador (2008). Constitución de la República del Ecuador. Quito: Asamblea Nacional del Ecuador.

BARR, R. R. (2017). The Resurgence of Populism in Latin America. Boulder. Boulder, EE. UU.: Lynne Rienner Publishers.

Basabe-Serrano, S., Martínez, J. (2014). Ecuador: cada vez menos democracia, cada vez más autoritarismo...con elecciones. Revista de Ciencia Política, 34(1), 145-170.

Basabe-Serrano, S., Pachano, S., A. Mejía Acosta. (2010). La democracia inconclusa: derechos fundamentales, instituciones políticas y rendimientos gubernamentales en Ecuador (1979-2008). Revista de Ciencia Política, 30(1), 65-85.

Boccagni, P., J. Ramírez. (2013). Building Democracy or Reproducing 'Ecuadoreanness'? A Transnational Exploration of Ecuadorean Migrants' External Voting. Journal of Latin American Studies, 45(4), 721-750.

CARreras, M. (2012). The rise of outsiders in Latin America, 1980-2010: An institutionalist perspective. Comparative Political Studies, 45(12), 1451-1482.

Castellanos, A. S., C. Ulloa. (2016). Representación política de las mujeres a nivel legislativo en Ecuador: un análisis entorno a la promoción de los derechos de la mujer desde 1941 a 2014. Revista de Ciencias Políticas y Relaciones Internacionales, 5(5), 8-29.

CNE (2014). Atlas Electoral 2002-2014. Quito, Ecuador: Consejo Nacional Electoral del Ecuador.

CNE (2021). Sistema Estadístico Electoral. Quito, Ecuador: Consejo Nacional Electoral del Ecuador 
Collins, J. (2014). New Left Experiences in Bolivia and Ecuador and the Challenge to Theories of Populism. Journal of Latin American Studies, 46(1), 59-86.

Collyer, M. (2014). Inside out? Directly elected 'special representation' of emigrants in national legislatures and the role of popular sovereignty. Political Geography, 41, 64-73.

Conaghan, C., C. De la Torre. (2008). The permanent campaign of Rafael Correa: Making Ecuador's plebiscitary presidency. The International Journal of Press/Politics, 13(3), 267-284.

Cyr, J. (2016). Between Adaptation and Breakdown: Conceptualizing Party Survival. Comparative Politics, 49(1), 125-145.

DANDOy, R. (2014). El desempeño electoral de los partidos políticos provinciales en las elecciones seccionales 2014 en Ecuador. Democracias, 2, 3-19.

Dandoy, R. (2017). Provincial dynamics in the 2017 national elections in Ecuador. Democracias, 5, 3-24.

DÁvila, D., K. N. Wylıe. (2021). Party Leadership and Institutionalization in Latin America. Oxford Research Encyclopedia in Latin American Politics. doi: https://doi.org/10.1093/acrefore/9780190228637.013.1667

Gallagher, K. P., A. Irwin, K. Koleski. (2013). ¿Un mejor trato?: Análisis comparativo de los préstamos chinos en América Latina. Ciudad de México, México: Universidad Nacional Autónoma de México, Facultad de Economía, Centro de Estudios China-México (CECHIMEX).

Freidenberg, F. (2006). Izquierda versus derecha. Polarización ideológica y competencia política en el sistema de partidos ecuatoriano. Política $y$ Gobierno, 13(2), 237-278.

FreidenberG, F. (2012). Ecuador 2011: Revolución Ciudadana, estabilidad presidencial y personalismo político. Revista de Ciencia Política, 32(1), 129-150.

Freidenberg, F. M. AlcÁntara-SÁez. (2001). Cuestión regional y política en Ecuador: partidos de vocación nacional y apoyo regional. Revista América Latina Hoy, (27), 123-152. doi: https://doi.org/10.14201/alh.2747

Freidenberg, F., S. Pachano. (2016). El sistema político ecuatoriano. Quito, Ecuador: FLACSO Ecuador. 
Herrera, E., S. Umpiérrez de Reguero, J. Chuya, C. Zurita, A. Palacio. (en prensa). Economía, actitudes políticas y comportamiento electoral: ¿una relación circular? En I. Ríos Rivera. y S. Umpiérrez de Reguero, S. (Eds.), Populismo y comportamiento político: incorporando la agenda ideacional. Guayaquil, Ecuador: Universidad Casa Grande.

Jara-Alba, C., T. LóPez-Guzman. (2015). México y Ecuador: un estudio comparativo de remesas e impacto macroeconómico. Revista Ciencia UNEMI, 8(15), 18-31.

Jara-Alba, C., S. Umpiérrez de Reguero. (2014). Evolución del sector público ecuatoriano de 1998 a 2013. Revista Enfoques: Ciencia Política y Administración Pública, 12(21), 131-148.

Labarthe, S., M. Saint-Upéry. (2017). Leninismo versus correísmo: la 'tercera vuelta' en Ecuador. Nueva Sociedad, 272, 29-42.

LAPOP. (2016). El Barómetro de las Américas por el Proyecto de Opinión Pública de América Latina (LAPOP): www.LapopSurveys.org.

Levitsky, S., J. Loxton. (2013). Populism and competitive authoritarianism in the Andes. Democratization, 20(1), 107-136.

Levitsky, S., J. Loxton, B. VAn Dyck (Eds.). (2016). Challenges of party-building in Latin America. Cambridge: Reino Unido: Cambridge University Press.

Ley de Elecciones (2000). Registro Oficial 117 del 11 de julio del 2000. Quito, Ecuador.

Ley Orgánica de Amparo Laboral de Mujeres (1997). Quito, Ecuador.

Ley Orgánica Electoral y de las Organizaciones Políticas: Código de la Democracia. (2009). Suplemento 578 de 27 de abril de 2009. Quito, Ecuador.

Luna, J. P., C. Rovira Kaltwasser. (2014). The Resilience of the Latin American Right. Baltimore, EE. UU.: The Johns Hopkins University Press.

Machado Puertas, J.C. (2007). Ecuador: el derrumbe de los partidos tradicionales. Revista de Ciencia Política, 27(especial), 129-147.

Machado Puertas, J.C. (2008). Ecuador...hasta que se fueron todos. Revista de Ciencia Política, 28(1), 189-215.

Mainwaring, S. (2006). The crisis of democratic representation in the Andes. Journal of Democracy, 17(3), 13-27.

MelÉndeZ, C. (2019). La derecha que se bifurca. Las vertientes populista-conservadora y tecnocrática-liberal en Perú post-2000. Colombia Internacional, (99), 3-27.

Meléndez, C., y Moncagatta, P. (2017). Ecuador: Una década de correísmo. Revista de Ciencia Política, 37(2), 413-448. 
Moncagatta, P., C. Espinosa Fernández de Córdoba (2019). No Country for Right Wing? La derecha ecuatoriana en el siglo XXI. Colombia Internacional, (99), 121-150.

Montúfar, C. (2016). ¿Vivimos en democracia?: análisis conceptual del régimen político de la revolución ciudadana. Quito, Ecuador: Corporación Editora Nacional: Universidad Andina Simón Bolívar.

Mustillo, T. J. (2007). Entrants in the political arena: new party trajectories during the third wave of democracy in Latin America. Doi: https://doi.org/10.17615/ m3hz-sz66

Mustillo, T. J., J. Polga-Hecimovich. (2018). Measures and votes: Party performance under free list proportional representation with evidence from Ecuador. Electoral Studies, 56, 124-135.

Nohlen, D. (2012). Gramática de los sistemas electorales. Una introducción a la ingeniería de la representación. Quito, Ecuador: Instituto de la Democracia del Consejo Nacional Electoral del Ecuador.

OrTiz, R. (2012). Sistema político y sistema electoral en Ecuador. Quito, Ecuador: Tribunal Contencioso Electoral del Ecuador.

Pachano, S. (2004). Proceso electoral de Ecuador. Quito, Ecuador: Flacso Ecuador.

Pachano, S. (2010). Ecuador: El nuevo sistema político en funcionamiento. Revista de Ciencia Política, 30(2), 297-317.

Pachano, S. (2012). Estado actual y futuro de la democracia en Ecuador. En A. Dargatz, M. Zouzo (Eds.). Democracias en transformación, ¿Qué hay de nuevo en los nuevos Estados Andinos? (pp. 85-102). Quito, Ecuador: Friedrich Ebert Stiftung (FES).

Palop-García, P. (2018). Contained or represented? The varied consequences of reserved seats for emigrants in the legislatures of Ecuador and Colombia. Comparative Migration Studies, 6(1), 38.

Polga-Hecimovich, J. (2013). Ecuador estabilidad institucional y la consolidación de poder de Rafael Correa. Revista de Ciencia Política, 33(1), 135-160.

RAmírez, J. (2018). Estado, migración y derechos políticos. El voto de los ecuatorianos desde el extranjero. Foro Internacional, 58(4), 755-804.

Rosenblatt, F. (2018). Party Vibrancy and Democracy in Latin America. New York, EE. UU.: Oxford University Press.

SÁnchEZ, F. (2008). ¿Democracia no lograda o democracia malograda?: un análisis del sistema político del Ecuador, 1979-2002. Quito, Ecuador: FLACso. 
Seawright, J. (2012). Party-system collapse. The Roots of Crisis in Peru and Venezuela. Stanford, EE. UU.: Stanford University Press.

Sotomayor Valarezo, P., S. Huertas-Hernández (2021). El camino hacia los gabinetes ministeriales: un estudio de los factores que influyen en la designación de mujeres ministras en Ecuador y Colombia, 1978-2018. Colombia Internacional, 105, 29-55.

UMPIÉRREZ de Reguero, S. (2016). Ecuador: entre la democracia y el autoritarismo. En. J. Olguín Olate (Ed.). Estados autoritarios latinoamericanos: Ayer, hoy y proyecciones (pp. 205-219). Santiago, Chile: Universidad Central.

Umpiérrez de Reguero, S., R. Dandoy. (2020). Extending the incumbency presence abroad. The case of MPAIS in Ecuadorian overseas district. En T. Kernalegenn y E. Van Haute (Eds.). Political Parties Abroad: A New Arena of Party Politics (115-132). London and New York, United Kingdom, EE. UU.: Routledge.

Umpiérrez de Reguero, S., R. Dandoy (2021). Should we go abroad? The strategic entry of Ecuadorian political parties in overseas electoral districts. Representation. doi: https://doi.org/10.1080/00344893.2021.1902850

Umpiérrez de Reguero, S., C. Jara-Alba, Cassis Jurado, A. (2016). Ecuador, Mujeres y Representación Legislativa. Revista Enfoques: Ciencia Política y Administración Pública, 14(24), 13-40.

Vera Rojas, S., S. Llanos-Escobar. (2016). Ecuador: La democracia después de nueve años de la 'Revolución Ciudadana' de Rafael Correa. Revista de Ciencia Politica, 36(1), 145-175.

Vommaro, G. (2019). De la construcción partidaria al gobierno: PRO-Cambiemos y los límites del 'giro a la derecha' en Argentina. Colombia Internacional, 99, 91-120.

Webb, P., S. Scarrow, T. Poguntke, (2019). Party organization and satisfaction with democracy: inside the blackbox of linkage. Journal of Elections, Public Opinion and Parties. doi: https://doi.org/10.1080/17457289.2019.1698047

Wolff, J. (2018). Ecuador after Correa: The struggle over the Citizens' Revolution. Revista de Ciencia Politica, 38(2), 281-302.

World Economic Forum. (2016). The Global Gender Gap Report 2016. Recuperado de: http://www3.weforum.org/docs/GGGR16/WEF_Global_Gender_Gap_ Report_2016.pdf

Los dos autores colaboraron de la misma manera para la elaboración y edición del manuscrito. El orden de la autoría es alfabético. 


\section{Anexo 1}

\begin{tabular}{|c|c|c|c|}
\hline \multicolumn{4}{|c|}{ Elecciones en Ecuador con candidaturas de CREO (2013-2021) } \\
\hline Elección (año) & Nivel de elección & Tipo de elección & $\begin{array}{l}\text { Participación de } \\
\text { candidatos CREO }\end{array}$ \\
\hline \multirow{5}{*}{$\begin{array}{l}\text { Elecciones } \\
\text { generales (2013) }\end{array}$} & \multirow{4}{*}{ Nacional } & $\begin{array}{l}\text { Ejecutivo } \\
\text { (primera vuelta) }\end{array}$ & Sí \\
\hline & & Ejecutivo (balotaje) & No aplica \\
\hline & & $\begin{array}{l}\text { Legislativa } \\
\text { (asientos nacionales) }\end{array}$ & Sí \\
\hline & & $\begin{array}{l}\text { Legislativo } \\
\text { (asientos provinciales) }\end{array}$ & Sí \\
\hline & $\begin{array}{l}\text { Supranacional } \\
\text { (Parlamento Andino) }\end{array}$ & Legislativo & Sí \\
\hline \multirow{4}{*}{$\begin{array}{l}\text { Elecciones sec- } \\
\text { cionales }(2014)\end{array}$} & Regionales & Ejecutivo & Sí \\
\hline & \multirow{2}{*}{ Local } & Ejecutivo & Sí \\
\hline & & Legislativo & Sí \\
\hline & Parroquial & Legislativo & Sí \\
\hline \multirow{5}{*}{$\begin{array}{l}\text { Elecciones } \\
\text { generales (2017) }\end{array}$} & \multirow{4}{*}{ Nacional } & Ejecutivo (primera vuelta) & Sí \\
\hline & & Ejecutivo (balotaje) & Sí \\
\hline & & $\begin{array}{l}\text { Legislativa (asientos na- } \\
\text { cionales) }\end{array}$ & Sí \\
\hline & & $\begin{array}{l}\text { Legislativo (asientos pro- } \\
\text { vinciales) }\end{array}$ & Sí \\
\hline & $\begin{array}{l}\text { Supranacional } \\
\text { (Parlamento Andino) }\end{array}$ & Legislativo & Sí \\
\hline \multirow{5}{*}{$\begin{array}{l}\text { Elecciones sec- } \\
\text { cionales }(2019)\end{array}$} & Nacional & $\begin{array}{l}\text { Consejo de Participación } \\
\text { Ciudadana y Control } \\
\text { Social }\end{array}$ & No aplica \\
\hline & Regionales & Ejecutivo & Sí \\
\hline & \multirow{2}{*}{ Local } & Ejecutivo & Sí \\
\hline & & Legislativo & Sí \\
\hline & Parroquial & Legislativo & Sí \\
\hline \multirow{5}{*}{$\begin{array}{l}\text { Elecciones gene- } \\
\text { rales }(2021)\end{array}$} & \multirow{4}{*}{ Nacional } & Ejecutivo (primera vuelta) & Sí \\
\hline & & Ejecutivo (balotaje) & Sí \\
\hline & & $\begin{array}{l}\text { Legislativa } \\
\text { (asientos nacionales) }\end{array}$ & Sí \\
\hline & & $\begin{array}{l}\text { Legislativo } \\
\text { (asientos provinciales) }\end{array}$ & Sí \\
\hline & $\begin{array}{l}\text { Supranacional } \\
\text { (Parlamento Andino) }\end{array}$ & Legislativo & Sí \\
\hline
\end{tabular}

Nota: se excluyó los referéndums y consultas populares a nivel nacional, regional y local.

Fuente: elaboración propia con base en los resultados electorales del CNE (2021) 
